\title{
Diabetic Foot Care in Patients with Mental Illness
}

\author{
Shinu Kuriakose* \\ School of Health Professions, New York Institute of Technology, Old Westbury, New York, USA \\ ${ }^{\star}$ Corresponding author: Dr. Shinu Kuriakose, School of Health Professions, New York Institute of Technology, Old Westbury, New York, USA; Email: skuria06@nyit.edu
}

Received: March 06, 2021; Accepted: March 12, 2021; Published: March 17, 2021

\begin{abstract}
Foot disorder pathologies, caused by diabetes mellitus, result in diminishing the quality of life for patients and straining the economic fabric of both governmental institutions and private organizations. Patients afflicted with diabetes suffer from disabilities, which can in many cases lead to foot amputation and other various complications. Often, patients also suffer from chronic pain caused by diabetic foot neuropathy, which may make them unable to continue with their employment and have a negative effect on their daily lives. Patients with mental health disorders are adversely affected by diabetic foot pathologies due to their lack of timely follow-up and limited access to medical providers. The goal of this Practicum experience was to develop and deliver a PowerPoint presentation to provide clinical guidance and education to primary care clinicians who evaluate diabetic patients (many with mental health issues) who otherwise might not have an opportunity to be evaluated by a podiatrist in a timely manner. This author created the PowerPoint presentation based on the review of literature and the input from the committee members. The PowerPoint presentation on diabetic foot care was limited to the primary care clinical staff at the Queens County Medical Center in Queens, New York. The purpose of the practicum is for these clinicians to learn how to perform a comprehensive evaluation of the lower extremities in patients with diabetes, including awareness of risk factors prone to increasing risk of foot ulceration, screening for neurological foot deficits, analyzing treatment options for patients with diabetic foot maladies, and exploring prevention strategies to prevent diabetic foot complications.
\end{abstract}

Keywords: Diabetic neuropathy, Diabetic foot care, Diabetic foot ulcer, Podiatry, Psychiatry and diabetes, Diabetes screening

\section{Introduction}

\section{Nature of the Problem}

Diabetes is a disorder, which can cause severe medical problems with the physiology of persons afflicted with it, and the silent nature of this malady can lead to medical disability in a manner where late intervention is futile. One of the greatest pathologies caused by diabetes is neuropathy in the peripheral lower limbs, which can cause not only physical injuries affecting mobility in patients but also issues with pain and poor healing, which can lead to chronic pain syndromes [1]. Diabetes occurs due to glucose not being properly regulated by either lack of insulin production by the pancreas or the inability of the body to use its own insulin in an effective manner. This process causes grave health consequences including lower-extremity amputations, a disease that is the seventh leading cause of death in the United States [2].

Patients, suffering from mental health problems, are especially harmed by diabetes as the lack of timely access to podiatric providers often leads to diabetic foot neuropathy. Neuropathy, which leads to loss of sensation in lower extremities of patients (due to the fact that these are longest nerves as they are involved in innervating all the way the bottom of the feet), can often cause ulcerations and foot sores, which are overlooked by these patients. The disorder of diabetic foot disease (DFD) is a combination of peripheral neuropathy and arterial disease, which often leads to an end result of foot ulceration and lack of appropriate management that can lead to lower extremity amputation complicated with an open wound infection or osteomyelitis [3]. Furthermore, this lack of foot malady awareness can cause these patients to not seek care for their feet and further compound these problems and, in many cases, lead to avoidable foot amputations. Additionally, these psychiatric patients face further barriers as they lack financial resources to see specialist clinicians promptly, are prone to increased risk by being on psychiatric medicines, which often increase their blood sugar levels, and lack proper housing and followup, which could enhance their medical care [4]. Proper screening, evaluation, and timely management and prevention of foot maladies in patients with diabetes have shown to significantly reduce the incidences of lower foot amputation and better health outcomes for diabetic patients [5].

\section{Significance to Patients with Mental Illness}

The provision of providing cutting-edge information to primary care practitioners, which includes screening, diagnosing, and treating psychiatric patients with diabetic fool maladies, facilitate the optimal goal of improving foot health care in this underserved population. The majority of mental health patients face challenges in seeing a podiatrist on a routine basis and thus, are at increased risk of diabetic foot pathologies due to the increased levels of hyperglycemia caused by psychiatric medications. The timely intervention of podiatric are provided by primary care clinicians will lead to positive health outcomes for patients afflicted with mental illness and improve their quality of life. 


\section{Review of Literature}

\section{Overview}

The Centers for Disease Control and Prevention (CDC) defines diabetes as a disorder, which leads to increased blood glucose levels in the body (CDC, 2015). Diabetes occurs due to glucose not being properly regulated by either lack of insulin production by the pancreas or the inability of the body to use its own insulin in an effective manner. This process causes grave health consequences, including lower-extremity amputations, a disease that is the seventh leading cause of death in the United States (CDC, 2015). According to the CDC, approximately 29.3 million people in the United States (9.3\% of the population) have diabetes with another 86 million people having a pre-diabetic condition (CDC, 2015). The incidence of diabetes mellitus is expected to increase from 382 million people in 2013 to 592 million by 2035 with the vast majority of cases present in developing countries, which will also see the greatest increase in the number of these cases [6]. It is ironic that as the world has developed exponentially in the fields of basic health care, disease and infection management, and improved diet for all in the last several decades, the elevated incidences of diabetes is a reminder of how overindulgence in this abundance of dietary choices and lack of exercise can lead to such a blood glucose malady [6].

\section{Diabetic Neuropathy}

DFD is a combination of peripheral neuropathy and arterial disease, which often leads to an end result of foot ulceration and the lack of appropriate management of DFD can lead to lower extremity amputations complicated with an open wound infection or osteomyelitis [3]. The World Health Organization (WHO) in conjunction with the International Diabetes Federation (IDF) have set a goal of reducing amputations related to diabetes complication by up to $50 \%$ by initiating a multipronged approach of increasing patient education about diabetes, forming a multidisciplinary team of various specialties involved with the patient, and timely patient monitoring, which has shown to decrease amputation rates from $49 \%$ to $85 \%$ [7]. Neuropathy leads to loss of sensation in the lower extremities of patients (due to the fact that the bottom of the feet are enervated by the longest nerves in the body) and can often cause ulcerations and foot sores, which are overlooked by these patients. Furthermore, this lack of foot malady awareness can cause these patients to not seek care for their feet and therefore further compound these problems; a tragic complication leading to avoidable foot amputations [3].

\section{Central Nervous System Effects}

Diabetes can cause significant damage to the organs of the central nervous system, primarily the brain. The increased levels of glucose present in the blood stream cross the blood-brain barrier leading to consequences, which include (a) encephalopathy due to problems metabolizing the increased glucose in the blood stream; (b) elevated risk of brain stroke due to elevated intracranial pressure as a result of chronic, uncontrolled diabetes; (c) potential of brain damage and white matter changes in the cerebral cortex due to the long term use of insulin and other hypoglycemic medications; (d) possibility of seizures due to elevated blood sugars decreasing the seizure threshold; and (e) potential of cognitive changes when hyperglycemia goes unchecked for long periods of time [8].

\section{Peripheral Nervous System Effects}

The effects of long-term elevated serum glucose levels have a deleterious effect on the nerves of the peripheral nervous system. The first effect, causing the most common nerve damage in diabetes mellitus, is called diabetic neuropathy, which impedes the sensation perceived by the sensory nerves as they relay messages to the brain [9]. Additionally, motor nerves are also affected as they slow down messages from the brain to move muscles in a timely manner affecting body reflexes to stimuli [10]. The second effect is autonomic neuropathy, which is a process whereby gastrointestinal functions such as digestion are affected by elevated blood sugar levels. Furthermore, autonomic involuntary functions such as blood pressure and heart pulse are often disrupted while also leading to sexual dysfunction (CDC, 2015). Another adverse effect is diabetic gastroparesis, which affects the passage of food through the stomach, as muscles in the colon do not interact in an effective manner, leading to multiple systemic medical complications [9]. Moreover, the process called focal diabetes-related neuropathy causes individual cranial nerves to transmit signals less effectively resulting in vision loss and hearing problems [9].

\section{Diabetic Foot Effects}

\section{Epidemiology}

There is an increased incidence of diabetes in the world, especially as the accessibility of medical care has improved [6]. The world population is living longer although enduring more diseases as people age with the latest estimate of 592 million people diagnosed with diabetes by 2035 [6]. Males, especially over the age of 60 , are at the highest risk of developing DFD with the presence of foot ulceration a major indicator leading to risk of foot amputation, a lifetime risk of almost 25\% prevalence in the general population [3]. The rate of foot ulceration increased by $700 \%$ in patients with diabetic neuropathy when compared with patients without diabetes and its associated conditions [3]. The National Institute of Diabetes and Digestive and Kidney Diseases (NIDDKD) estimated that about $60 \%$ to $70 \%$ of the world population with diabetes suffers from neuropathy (age and disease duration causing increased risk) and the highest rates are among people with diabetes for over 25 years [11].

\section{Etiology}

The lack of knowledge regarding this disease, unable to see providers on time, lack of screening protocols, difficulty in managing blood sugar levels, and the limited follow-up options available for patients often led to this result of foot amputation [3]. The loss of sensation due to sensory nerves being initially unresponsive to light stimuli makes the patients unaware of any stressors affecting their lower extremities, which often results in foot injuries being unheeded. Gradually, this loss of sensory stimuli perception ascends into more proximal areas of both feet bilaterally, and by then, most of the damage has been done in terms of nerve neuropathy. Often, patient complaints gradually evolve from tingling sensations and shock-like lower extremity pain to abnormal hot 
and cold sensation to their feet; a process that eventually leads to foot cramping with anatomic changes like hammer toes, flat feet, and foot pressure ulcerations [3]. These anatomic results are directly from motor nerves being affected during the later phases of DFD after initial sensory foot deprivation. Another common malady leading to foot dysfunction includes autonomic changes in the feet, which can often manifest itself as dry skin, cracked skin changes, excessive foot sweating at times, and loss of deep tendon reflexes. The difficulty of blood to flow back from the veins in the feet against gravity leads to foot edema, which is another major risk factor of DFD [3].

\section{Risk Factors}

There are various risk factors which can make an individual prone to be diagnosed with diabetes mellitus relative to the general population. Family history plays a significant part in elevating a person's chance of getting diabetes. The elevated risk can be as high as 2.3 to 5.5 times a person without a familial history of diabetes [12]. Multiple other risk factors include obesity, waist circumference, inactivity, and history of gestational diabetes. Additionally, American Indians, Hispanics, and Asians were noted to have elevated risk of being diagnosed with diabetes compared to the general population [10]. It was also noted that losing muscle mass, co-morbid medical conditions, and gaining weight due to inactivity also led to increased prevalence of diabetes in the older population [10]. High blood pressure, increased triglycerides, and elevated cholesterol levels also were noted to be risk factors in developing this illness [12].

\section{Oral Medications}

There are multiple medications used to mitigate the adverse effects of diabetes by attempting to keep blood sugar levels down with the goal being to prevent long term central and peripheral manifestations of this disease. The majority of these classes of drugs include metformin, sulfonylureas, thiazolidinediones, meglitinides, dipeptidyl peptidase inhibitors, and glucagon receptor agonists, which are used as monotherapy and in combination, to treat adults with type 2 diabetes [13]. There was an average of $1 \%$ decline in Hemoglobin A1c (an indicator of blood sugar levels over the last three months) with these prescribed medications and there was no difference between a single drug or a combination.

\section{Lifestyle}

Patients with diabetes must adhere to a lifestyle, which includes awareness of risk factors prone to increasing their risk of foot ulceration, be aware of self-screening for neurological foot deficits, speak to their clinicians proactively regarding treatment options for diabetic foot maladies, and employ recommended prevention strategies to prevent diabetic foot complications [14]. Additionally, patients must be encouraged to ensure compliance with foot care protocols and adherence to follow-up recommendations and appointments [14].

\section{Diagnosis}

\section{History}

A prominent risk factor, which leads to adverse effects from diabetes is the asymptomatic nature of this illness in its early stage.
Patients are often not aware of their hyperglycemic status until they start experiencing symptoms. Early detection of diabetes leading to initiation of diet and exercise regimens with possible utilization of oral hypoglycemics has positive outcomes for patients. The signs and symptoms of diabetes include: (a) lethargy, (b) chronic feelings of hunger, (c) excessive urination with corresponding feelings of being thirsty most of the time, (d) dry skin, and (e) blurred vision [15]. Patients with diabetes are also prone to yeast infections due elevated blood sugars providing a nutritional source for yeasts and due to their immunocompromised status. Nerve damage associated with neuropathy can cause long duration for injuries to heal while also causing numbness and tingling in extremities. It takes a combination of factors, such as the patient history, family history, clinical foot exam, and the utilization of diagnostic exams, to best screen the patient for severity of DFD [15].

It is imperative that focused questions need to be asked during the history taking portion of evaluation which would include (a) is the patient's blood sugar monitored by the patient on an ongoing basis and is it well controlled; (b) are the medications taken by the patient for diabetes causing any adverse side effects including episodes of hypoglycemia; (c) has the patient been referred to podiatrists, ophthalmologists, nephrologists to evaluate and manage systemic issues caused by their diabetes; (d) has the patient been involved in their diet management by exercising and eating in a health manner; (e) has the patient kept up-to-date with their immunizations including annual flu vaccine shots; and (f) has the patient made aware of all foot complications caused by diabetes and has the patient been educated in foot care, including keeping the foot area dry, examining the foot area on a regular basis for any bruises or injuries and ensuring that their toe nails are trimmed regularly by a podiatrist [3].

\section{Physical Exam}

The physical exam component of the evaluation of a patient with diabetes must ensure that the patient's body mass index (BMI) is maintained in the normal range while also ensuring that the patient maintains a consistent exercise regimen (NIDDKD, 2008). Vital signs must be monitored for hypertension and orthostatic blood pressure must be performed regularly to rule out signs of autonomic neuropathy [7]. Physical exam techniques must also evaluate the foot of the patient regularly for injuries and pulses must be palpated to monitor for adequate blood flow to the extremities [7]. A combination of deep tendon reflexes, sensory lower extremity exams, and vibration tests must be performed to evaluate patient for any metabolic foot maladies [3]. An ophthalmology exam must be performed to rule out neo-vascularization in the retinal region and flame hemorrhages which are often noted in diabetic patients with poor blood sugar control (NIDDKD, 2008). A thorough skin examination must be performed to screen for skin atrophy, dryness, yeast infections and discoloration of the skin, which if often noted in diabetic patients [4].

\section{Lab Studies}

There are multiple scales, which can be used to measure foot maladies, and they might utilize measures, such as capillary refill, pedal pulses, foot deep tendon reflexes, pathological Babinski 
reflexes, and pedal edema on rating scales [15]. Commonly used screening diagnostic methods, such as neuropathy symptoms scores, Semmes-Weinstein monofilament, vibration perception on the lower extremities from distal to proximal, nerve conduction studies, and even nerve biopsy, are valid tools in the arsenal for diabetic neuropathy foot screenings [15].

\section{Treatment}

The mainstay of treatment is a coordinated approach by the patients and their clinicians regarding prompt screening, initial diagnosis of diabetes and subsequent awareness of systemic foot manifestations, and monitoring levels of blood sugars, ensuring that the foot is free of any added physical stress by managing weight and reducing risk factors, such as smoking and alcohol use and appropriate follow up with referral specialists [3]. A combination of clinicians would include the primary care clinician to coordinate care and refer to specialists, dietician to monitor weight and recommend food choices, diabetes educator who might suggest special techniques to care for the foot [3]. Additionally, an endocrine specialist is needed to follow up on glycemic control and management of medications, a podiatrist to ensure that the foot is evaluated thoroughly and recommend and fit podiatric shoes for the patient if necessary, and family support to help ensure that the patient is able to follow through with all his or her medical commitments [3].

\section{Primary Interventions}

In case of an open ulcer, there would be certain recommended treatments, which are considered standard protocols to include (a) debridement of the wound to help remove any foreign bodies and ensure that the food is free of infection; (b) changing the pressure points of the foot by utilizing podiatric foot inserts and making special leg casts as necessary; and (c) treatment of any infection by using topical and/or oral antibiotic agents to reduce opportunities for any spread of infection, which could lead to cellulitis and gangrene if unchecked [3]. Wound dressings also play a key role in maximizing the role of antibiotics and reducing chances of infection in patients with diabetic foot ulcers [3]. Common wound dressing techniques include wet-to-dry saline dressings; dressings with infused topical antibiotics covering the wound; and polyurethane dressings, which help ensure that the moisture conditions are optimal for wound healing [3]. Increasingly, there is greater use of growth factors in the treatment of diabetic foot ulcers that help with angiogenesis and increased cellular production, which can lead to more vascularization of the site. Furthermore, the role of vacuum-assisted closure, which can help remove redundant moisture in the wound, exposes wounds to rich oxygenated air therapy, and stem therapy is being studied in the quest to manage and treat diabetic foot complications [3].

\section{Complications}

Another common complication of diabetic neuropathy is peripheral artery disease (PAD), which manifests itself in the lower extremities by chronic leg pain, difficulty with healing of foot wounds, change in foot temperature, and lack of foot pulses bilaterally [16]. Commonly used screening tools for PAD include measuring the ankle-brachial index, foot ultrasounds, and contrast angiography [16]. Although these tools continue to be prevalent and utilization of these techniques have increased over the last several years, there continues to be increased rates of diabetic foot amputations due to a lack of timely screening and access to providers [3].

\section{Prevention}

The vast number of patients with diabetes are not initially seen by a podiatrist but rather by their primary care providers, including allied health professionals (physician assistants), and preventive strategies, screening, and treatment of foot ulcers has shown to decrease lower foot amputation rates by $49 \%$ to $85 \%$ (NIDDKD, 2008). Physician assistants (PAs) can play a crucial role in mitigating the amputations connected with poor foot hygiene in diabetics by ensuring that their diabetic patients are screened in an appropriate fashion during their initial visit with an emphasis on thorough foot screening and followup referral to a podiatrist if available in a timely fashion (NIDDKD, 2008).

\section{Patient Education}

The screening criteria for DFD must include initial awareness and identification that a patient is vulnerable for foot ulcerations due to the diagnosis of diabetes. The patient must be made aware that routine inspection of the foot on a daily basis (while in the shower for example) is essential to prevent damage to the foot, shedding light on the symptoms to look out for in DFD, such as temperature changes in the foot, tingling, and painful feet [15]. It is important that the patient wears protective shoes, which helps reduce stress on specific parts of the foot and reduces exposure of the foot to outside risk factors, leading to foot trauma and keeping an eye out for any calluses, hammertoes, or hardening of certain areas of the foot [15]. There also needs to be regular follow-up by patients with their clinicians, depending on the nature of their DFD, which might range from a yearly foot examination to seeing a clinician every couple of months if there is already an ulceration present [15].

Patients and their families must be educated about checking that the shoes they wear do not provide excessive stress to the feet, provide adequate cushioning in their daily walking shoes, check daily between their toes for ulcerations, wash and then thoroughly dry their feet daily before bed, and use precaution if utilizing tools to cut toenails [15]. Active self-care and awareness of diabetic manifestation in feet, especially being vigilant in the presence of foot lesions and ulcerations, leads to positive long-term health outcomes for patients with diabetes [14]. The recommendations for foot care, which showed a potential decrease of lower foot amputations by almost $85 \%$, included (a) always walking with comfortable shoes and not barefoot, (b) trimming toenails and not using abrasive items on feet, and (c) routine foot exams by a trained foot clinician [14-22].

\section{Topics Based on the Literature}

This author reviewed multiple research studies regarding the field of diabetic foot problems and the associated screening, diagnosis, treatment, and prevention protocols. It was imperative that the information regarding how prevalent diabetes is was included in the 
literature review of the topic, while also emphasize the frequency in which amputations occur in diabetic patients who neglect foot care [3]. The review of literature, after briefly touching on the definition of diabetes and related causes, focused on diabetic foot disorders. It was emphasized in the practicum review that screening and early diagnosis of diabetes can play a crucial role in reducing the frequency and intensity of foot infections [2].

The overarching theme of the practicum topics incorporated in the review of literature focused on complications of diabetes on a systemic manner, which primarily focused on the foot in this report. The importance of early intervention, including treatment with medication and emphasis of patient education, was dwelt upon as a compelling factor in decreasing the incidence of diabetic foot maladies. The author also emphasized the importance of referring patients to follow-up care with specialists due to the nature of diabetic disease and provided information for the primary clinician to implement treatment protocols during this initial treatment phase [15]. Finally, the review of literature accentuated the fact that early prevention, screening, treatments, and robust patient education would empower patients to take decisive steps in their diabetic foot care leading to positive health outcomes.

\section{Discussion}

Diabetic foot disorders can play a prominent role in affecting mobility in patients afflicted with this disease, which can often result in chronic pain and limited healing in the lower extremities [1]. The lack of insulin production by the pancreas in diabetic patients often lead to foot amputations, and this process is the one of the leading causes of death in the United States [9]. A combination of factors including lack of proper screening, timely evaluation, appropriate management and limited patient education work in conjunction to exacerbate this condition leading to poor patient outcomes [5]. Moreover, patients with limited financial and social resources can suffer additional negative consequences of this disease compared to the regular population due to their socio-economic circumstances [3].

Patients with mental health disorders encounter additional challenges due to psychiatric medications increasing blood sugar levels which further exacerbate their diabetic condition while also negotiating lack of proper housing and follow-up medical care [4]. This author focused on the barriers, which economically deprived patients face regarding diabetic care and the opportunities available to overcome them. The limited access to specialist foot providers, such as podiatrists, made it imperative that primary care clinicians, who after are the first to evaluate patients for medical purposes be educated in initial screening and management of diabetic foot maladies [7]. This author developed a PowerPoint presentation which was delivered to a group of primary care clinicians at the Queens County Medical Center, located in Queens, New York, which is at the front line of delivering care to indigent and socially deprived populations. It was the goal of this presentation that these primary care clinicians would be educated to treat patients early enough in the progression of diabetic foot disorders that diabetic related foot amputations will be minimized; a process leading to improved quality of life for these patients [5].

\section{Conclusion}

Lower extremity amputations lead to a decreased quality of life issues for patients with associated economic challenges and mobility issues. Timely screening, management, patient education, and appropriate follow-up can lead to mitigating the effects of diabetes not only on foot related problems, but on the overall health of patients. Patient education in conjunction with primary care provider education can play a crucial role in minimizing the devastating effects of this disease and lead to better health outcomes for patients, and reducing the financial expenses in the long term to the United States health care budget.

Appropriate and timely management of diabetes with its associated morbidities can lead to positive health outcomes for patients. The devastating consequences of diabetic related foot amputations not only leads to a poorer quality of life for affected patients but has caused increased economic harm to the community. Provider education regarding early screening and treatment of diabetes, which will lead to appropriate referrals to specialists, can help all the stakeholders affected by this disease on a personal and professional level. Governmental agencies, private institutions, and insurance companies will certainly benefit economically if there are more resources expended in the early phases of screening and patient education of diabetic patients. Finally, patients afflicted with this disorder would be able to be productive members of society without suffering the consequences of long-term foot pain and diabetic amputations.

\section{Recommendations}

Exploration of the diabetic foot disorders presents an opportunity for front line primary care clinicians to be leaders in the quest to reduce diabetic foot related morbidities. This innovative process would help enhance both patient and provider satisfaction while also providing economic savings to these institutions, which otherwise would be burdened by diabetic foot-related expenses. Further research could encompass patients not only suffering from psychiatric disorders, but also (a) patients with alcohol and substance abuse disorders, (b) native American and inner city populations without access to timely medical care, and (c) disabled patients who are unable to seek professional help due to mobility issues. Additional funding grants by governmental organizations and private charities to explore opportunities to expand diabetic care would certainly enhance research into this important topic. Finally, recognition about diabetic foot disorders and its horrendous consequences on patients can help pique further interest in researchers to explore alternative opportunities to improve patient care and provide a valuable service to society.

\section{References}

1. Lazzarini P, Gurr J, Rogers J, Schox A, Bergin S. (2012) Diabetes foot disease: The Cinderella of Australian diabetes management? Journal of Foot and Ankle Research 5: 24. [crossref]

2. Centers for Disease Control and Prevention (2015, December 28) Basics about diabetes: What is diabetes? Retrieved from http://www.cdc.gov/diabetes/basics/ diabetes.html.

3. Amin N, Doupis J (2016) Diabetic foot disease: From the evaluation of the "foot at risk" to the novel diabetic ulcer treatment modalities. World Journal of Diabetes 7: 153-164. 
4. Hoffman K (2016, March 21) Managing lower extremity conditions in patients with mental illness. Podiatry Today 29.

5. Hsu CR, Chang CC, Chen YT, Lin WN, Chen MY (2015) Organization of wound healing services: The impact on lowering the diabetes foot amputation rate in a tenyear review and the importance of early debridement. Diabetes Research and Clinical Practice 109: 77-84. [crossref]

6. Guariguata L, Whiting DR, Hambletonc I, Beagleya J, Linnenkampa U, et al. (2014) Global estimates of diabetes prevalence for 2013 and projections for 2035. Diabetes Research and Clinical Practice, 103: 137-149. [crossref]

7. Bakker K, Apelqvist J, Schaper NC, International Working Group on the Diabetic Foot Editorial Board (2012) Practical guidelines on the management and prevention of the diabetic foot 2011. Diabetes Metabolism Research and Review, 28: 225-231. [crossref]

8. Prasad S, Sajja R, Naik P, Cucullo C (2014) Diabetes Mellitus and Blood-Brain Barrier Dysfunction: An Overview. Journal of Pharmacovigilance 02: 125. [crossref]

9. Tesfaye S, Selvarajah D (2012) Advances in the epidemiology, pathogenesis and management of diabetic peripheral neuropathy. Diabetes/Metabolism Research and Reviews 28: 8-14. [crossref]

10. The Mayo Clinic (2016, September 25) Diabetes-Risk Factors. Retrieved from http:// www.mayoclinic.org/diseases-conditions/diabetes/basics/risk-factors/con-20033091

11. National Institute of Diabetes and Digestive and Kidney Diseases. (2016, June 18) Diabetic neuropathies: The nerve damage of diabetes. Retrieved from http://www. niddk.nih.gov/health-information/health-topics/Diabetes/diabetic-neuropathiesnerve-damage-diabetes/Pages/diabetic-neuropathies-nerve-damage.aspx\#what

12. Chan JC, Malik V, Jia W, Kadowaki T, Yajnik CS, et al. (2009) Diabetes in Asia. The Journal of the American Medical Association 301: 2129.
13. Bennett WL, Maruthur NM, Singh S, Segal JB, Wilson LM, et al. (2011) Comparative Effectiveness and Safety of Medications for Type 2 Diabetes: An Update Including New Drugs and 2-Drug Combinations. Annals of Internal Medicine 154: 602. [crossref]

14. Bonner T, Foster M, Spears-Lanoix E (2016) Type 2 diabetes-related foot care knowledge and foot self-care practice interventions in the United States: A systematic review of the literature. Diabetic Foot \& Ankle 7: 29758. [crossref]

15. Lechleitner M, Abrahamian H, Francesconi C, Sturm W, Kohler G (2016) Diabetischer FuB. Wiener Klinische Wochenschrift 128: 80-84.

16. Beaney A, Nunney I, Gooday C, Dhatariya K (2016) Factors determining the risk of diabetes foot amputations-A retrospective analysis of a tertiary diabetes foot care service. Diabetes Research and Clinical Practice 114: 69-74. [crossref]

17. Bartsch RA, Cobern KM (2003) Effectiveness of PowerPoint presentations in lectures. Computers \& Education 41: 77-86.

18. Boghossian P (2006) Behaviorism, constructivism, and Socratic pedagogy. Educational Philosophy and Theory 38: 713-722.

19. Castillo M (2010) Making a Point: Getting the Most Out of PowerPoint. American Journal of Neuroradiology 32: 217-219.

20. Diabetes. (n.d.) In Merriam-Webster's online dictionary (11th ed.) Retrieved from http://www.merriam-webster.com/medical/diabetes

21. National Institute of Diabetes and Digestive and Kidney Diseases. (2016, June 18) Diabetic neuropathies: The nerve damage of diabetes. Retrieved from http://www. niddk.nih.gov/health-information/health-topics/Diabetes/diabetic-neuropathiesnerve-damage-diabetes/Pages/diabetic-neuropathies-nerve-damage.aspx\#what

22. Neuropathy (n.d.) In Merriam-Webster's online dictionary (11th ed.) Retrieved from http://www.merriam-webster.com/medical/neuropathy 